\title{
O papel do enfermeiro na classificação de risco nos serviços de urgência e emergência
}

\section{Nurse's role in the risk classification on emergency services}

\author{
Adrieli dos Santos Quaresma'• Daiani Modernel Xavier² • Marta Regina Cezar-Vaz ${ }^{3}$
}

\section{RESUMO}

Objetivou-se conhecer a atuação do enfermeiro na classificação de risco dos serviços de urgência e emergência. Trata-se de uma revisão integrativa, realizada a partir de consultas nas bases de dados LILACS, MEDLINE e BDENF, em artigos redigidos em inglês, espanhol e português, no recorte temporal de 2008 a 2018 . Foram selecionados 18 artigos, por meio de leitura crítica dos textos na íntegra. Os dados foram analisados através de estatística descritiva e Análise de Conteúdo. Verificou-se que dos 18 artigos analisados, nove (50\%) foram indexados na base de dados MEDLINE, seis (33,3\%) na LILACS e três (16,7\%) na BDENF. Percebeu-se variação de uma a três publicações por ano, com exceção dos anos 2009 e 2014 , em que não houve publicações. Após Análise de Conteúdo, identificaram-se três categorias: o enfermeiro no contexto da classificação de risco, capacitação do enfermeiro atuante na classificação de risco e a atuação do enfermeiro na classificação de risco. Concluiu-se que o enfermeiro da classificação de risco dos serviços de urgência e emergência participa na assistência, tomada de decisões, monitoramento dos pacientes, registros, gerenciamento, educação e resolubilidade dos problemas e/ou encaminhamento dos pacientes aos serviços adequados. Espera-se contribuir para melhor compreensão acerca da atuação do enfermeiro nesses ambientes.

Descritores: Enfermagem em Emergência; Papel do Profissional de Enfermagem; Triagem; Serviço Hospitalar de Emergência; Enfermagem.

\begin{abstract}
The objective of this study was to know the nurse's roles in the risk classification of the emergency departments. An integrative review carried out on LILACS, MEDLINE and BDENF, in articles written in English, Spanish and Portuguese, from 2008 to 2018 . Eighteen articles were selected through critical and integral reading of the text. Data were analyzed through descriptive statistics and Content Analysis. It was verified that of the 18 analyzed articles, nine (50\%) were indexed in the MEDLINE database, six (33.3\%) in LILACS and three (16.7\%) in BDENF. It was found a variation from one to three publications per year, except for the years 2009 and 2014, in which there were no publications. After Content Analysis, three categories were identified: the nurse in the context of the risk classification, the nurse's performance in the risk classification and the nurse's training in the risk classification. It was concluded that nurses acting in risk classification of emergency departments participate in care, decision making, patient knowledge, records, management, education and problem solving and/or referral of patients to the appropriate services. It is hoped to contribute to a better understanding of the nurse's performance in these places.
\end{abstract}

Keywords: Emergency Nursing; Nurse’s Roles;Triage; Emergency Department; Nurse.

'Acadêmica do Curso de Graduação em Enfermagem da Universidade Federal do Rio Grande (FURG), Rio Grande (RS), Brasil. E-mail: theadriquaresma@gmail. com

${ }^{2}$ Enfermeira. Doutora em Enfermagem. Docente do Curso de Graduação em Enfermagem da FURG, Rio Grande (RS), Brasil. E-mail: daiamoder@gmail.com ${ }^{3}$ Enfermeira. Doutora em Filosofia da Enfermagem. Docente do Curso de Graduação e do Programa de Pós-Graduação em Enfermagem da FURG, Rio Grande (RS), Brasil. E-mail: cezarvaz@vetorial.net 


\section{INTRODUÇÃO}

O objeto deste estudo são as evidências científicas acerca da atuação do enfermeiro na Classificação de Risco (CR) dos Serviços de Urgência e Emergência (SUE). Os SUE são ambientes dinâmicos e com ritmo acelerado de trabalho. $O$ objetivo desses ambientes é o fornecimento de intervenções e cuidados rápidos para indivíduos que apresentam quadros clínicos de trauma e doenças agúdas'. No entanto, existe a procura desses serviços como fonte alternativa à assistência ambulatorial, levando ao atraso no atendimento aos pacientes graves e à superlotação dos SUE².

A superlotação em SUE é um problema global. Mostra-se motivo de preocupação para os gestores, devido à maior incidência de eventos adversos ao paciente, atrasos no tratamento, altas taxas de mortalidade, prolongamento do período de internação e readmissão hospitalar ${ }^{3,4}$. Além disso, está associada à sobrecarga de trabalho para os profissionais de saúde e aumento no tempo de tomada de decisão clínica ${ }^{5}$.

Nesse contexto, no ano de 2004, foi implementado no Brasil o acolhimento com CR nos SUE, visando a redução da superlotação e suas consequências ${ }^{6}$. A CR é um processo que permite a gestão dos riscos clínicos, de modo que seja priorizado o atendimento e o tratamento dos pacientes que se encontram em situações críticas e sensíveis ao tempo ${ }^{7}$. É operacionalizada por meio da aplicação de escalas ou protocolos de CR, baseados na avaliação clínica do estado geral dos pacientes ${ }^{8}$.

A maioria dos protocolos nacionais fundamenta-se nos modelos internacionais Australasian Triage Scale (ATS) ou Manchester Triage System (MTS) ${ }^{9}$. Esses propõem a classificação dos pacientes em cinco níveis de urgência e a determinação de um tempo máximo de espera para cada categoria, a partir da avaliação de vias aéreas, respiração, circulação e estado de consciência9-11.

No âmbito da equipe de enfermagem, a CR e a priorização da assistência nos SUE é uma atividade privativa do enfermeiro, devendo ser realizada por meio do Processo de Enfermagem (PE). Para a operacionalização da CR, o enfermeiro necessita conhecimentos clínicos e habilidades técnicas, gerenciais e de raciocínio, a fim de garantir o rigor técnico-científico necessário à atividade ${ }^{12-13}$.

Diante do exposto, percebe-se a importância do enfermeiro neste processo. Nesse sentido, objetiva-se conhecer a atuação do enfermeiro na CR dos SUE. O estudo justifica-se pela necessidade de compreender a atuação do enfermeiro neste ambiente, a fim de que se identifiquem ações imprescindíveis do profissional e lacunas existentes no processo de CR, de forma a melhorar a assistência de enfermagem.

\section{MÉTODO}

Trata-se de uma revisão integrativa da literatura, cuja finalidade é reunir e sintetizar informações, teóricas ou empíricas, contidas na literatura, de maneira sistemática e ordenada, contribuindo para o aprofundamento do conhecimento a respeito do tema investigado. Esse método de revisão possibilita uma compreensão holística abrangente de problemas relevantes para os cuidados e políticas de saúde, contribuindo, consequentemente, para a melhoria da assistência à saúde ${ }^{14}$.

Para operacionalização desta revisão, utilizaram-se as seguintes etapas: formulação do problema, coleta de dados, avaliação dos dados, análise e interpretação dos dados coletados e apresentação dos resultados ${ }^{14}$.A etapa de formulação do problema resultou na questão norteadora: Como atua o enfermeiro na CR dos SUE?

A etapa de coleta dos dados aconteceu no mês de fevereiro de 2018. Procedeu-se a busca de pesquisas nacionais e internacionais indexadas nas bases de dados eletrônicas da Literatura Latino-Americana e do Caribe em Ciências da Saúde (LILACS), Literatura Internacional em Ciências da Saúde (MEDLINE) e Base de Dados em Enfermagem (BDENF), por meio de consultas na Biblioteca Virtual em Saúde (BVS) e na Pubmed. As buscas nas bibliotecas virtuais foram realizadas em dois computadores diferentes, simultaneamente, por dois pesquisadores, localizados em ambientes distintos e sem que houvesse comunicação entre eles, visando a garantia de dados fidedignos. Utilizaram-se palavras-chave separadas pelos operadores boleanos and e or, resultando na seguinte combinação:"Enfermagem" [or] "enfermeiro" [and] "triagem" [or] "classificação de risco" [and] "urgência e emergência". Dessa etapa adviram 98 estudos.

$\mathrm{Na}$ etapa de avaliação dos dados, procedeu-se à leitura de títulos e resumos dos 98 estudos. Foram utilizados os seguintes critérios de inclusão: artigos que respondessem a questão norteadora; publicados no período de 2008 a 2018; com texto disponível na íntegra e redigidos em português, espanhol ou inglês. Foram excluidos os estudos: que não responderam à questão norteadora, duplicados nas bases de dados, com texto indisponível na íntegra, resumos de congressos, anais, editoriais, monografias, dissertações e teses. A amostra final para análise foi composta de 18 artigos.

Para a análise dos dados, foi realizada a leitura crítica dos artigos na íntegra. Com a finalidade de comparar os dados, foi elaborado um instrumento contemplando as seguintes variáveis: título, autores, periódico de publicação, ano de publicação e objetivos do estudo. Após a leitura e tabulação dos dados, procedeu-se a caracterização dos artigos analisados através de estatística descritiva e a Análise de Conteúdo ${ }^{15}$ dos dados qualitativos. Os resultados foram apresentados por meio de estatística descritiva e a partir das categorias formuladas na etapa anterior.

Quanto aos aspectos éticos, este estudo foi realizado 
a partir de artigos nacionais e internacionais, cujos autores foram referenciados em todo o momento que seus artigos foram citados. Na revisão integrativa, é dispensada a submissão do estudo ao Comitê de Ética em Pesquisa, visto que os dados manipulados são de livre acesso, não se tratando de documentos em requeiram sigilo ético.

\section{RESULTADOS}

Nesta revisão foram analisados 18 artigos em consonância com os critérios de inclusão previamente estabelecidos. $\bigcirc$ quadro I apresenta a distribuição dos artigos de acordo com o título, os autores, o periódico de publicação, o ano de publicação e os principais objetivos.

QUADRO 1 - Especificações dos artigos científicos utilizados nesta revisão. Rio Grande (RS), Brasil, 2018.

\begin{tabular}{|c|c|c|c|c|c|}
\hline $\mathbf{N}^{\circ}$ & Título & Autores & Periódico & Ano & Objetivos \\
\hline 1 & $\begin{array}{c}\text { Manchester triage system in pediatric } \\
\text { emergency care: prospective } \\
\text { observational study }\end{array}$ & $\begin{array}{c}\text { Veen, MV; } \\
\text { Steyerberg, EW; Ruige, M; } \\
\text { Meurs, AHJV; } \\
\text { Lei, JVD; } \\
\text { Moll, HA. } \\
\end{array}$ & BMJ & 2008 & $\begin{array}{c}\text { Validar o uso do Sistema } \\
\text { de Triagem de Manchester } \\
\text { em cuidados de emergência } \\
\text { pediátricos }\end{array}$ \\
\hline 2 & $\begin{array}{c}\text { El triaje: herramienta fundamental en } \\
\text { urgencias y emergencias }\end{array}$ & $\begin{array}{l}\text { Soler, W; } \\
\text { Muñoz, MG; } \\
\text { Bragulat, E; } \\
\text { Álvarez, A. }\end{array}$ & $\begin{array}{l}\text { Anales del Sistema } \\
\text { Sanitario de Navarra }\end{array}$ & 2010 & $\begin{array}{c}\text { Refletir a respeito da } \\
\text { importância da triagem como } \\
\text { uma ferramenta em urgência e } \\
\text { emergência }\end{array}$ \\
\hline 3 & $\begin{array}{c}\text { Caracterização do atendimento após } \\
\text { implantação do acolhimento, avaliação } \\
\text { e classificação de risco em hospital } \\
\text { público }\end{array}$ & $\begin{array}{l}\text { Rossaneis, MA; } \\
\text { Haddad, MDCL; } \\
\text { Borsato, FG; } \\
\text { Vannuchi, MO; } \\
\text { Sentone, ADD. }\end{array}$ & $\begin{array}{l}\text { Revista Eletrônica de } \\
\text { Enfermagem }\end{array}$ & 2011 & $\begin{array}{l}\text { Descrever a implantação do } \\
\text { acolhimento com avaliação } \\
\text { e classificação de risco em } \\
\text { um hospital público de média } \\
\text { complexidade e caracterizar o } \\
\text { atendimento após a implantação } \\
\text { dessa estratégia }\end{array}$ \\
\hline 4 & $\begin{array}{l}\text { Be careful with triage in emergency } \\
\text { departments: interobserver agreement } \\
\text { on } 1578 \text { patients in France }\end{array}$ & $\begin{array}{c}\text { Durand, AC; } \\
\text { Gentile, S; } \\
\text { Gerbeaux, P; } \\
\text { Alazia, M; } \\
\text { Kiegel, P; } \\
\text { Luigi, S; } \\
\text { Lindenmeyer, E; } \\
\text { Olivier, P; } \\
\text { Hidoux, MA; } \\
\text { Sambuc, R. }\end{array}$ & $\begin{array}{l}\text { BMC Emergency } \\
\text { Medicine }\end{array}$ & 2011 & $\begin{array}{l}\text { Medir a concordância entre } \\
\text { os profissionais de saúde da } \\
\text { classificação de risco de um } \\
\text { departamento de emergência }\end{array}$ \\
\hline 5 & $\begin{array}{l}\text { Impact of the ABCDE triage in primary } \\
\text { care emergency department on the } \\
\text { number of patient visits to different parts } \\
\text { of the health care system in Espoo City }\end{array}$ & $\begin{array}{l}\text { Kantonen, J; } \\
\text { Menezes, R; } \\
\text { Heinänen, T; } \\
\text { Mattila, J; } \\
\text { Mattila, KJ; } \\
\text { Kauppila, T. }\end{array}$ & $\begin{array}{l}\text { BMC Emergency } \\
\text { Medicine }\end{array}$ & 2012 & $\begin{array}{c}\text { Avaliar se o fluxo de pacientes } \\
\text { foi alterado pela implementação } \\
\text { do sistema de triagem ABCDE } \\
\text { nos departamentos de } \\
\text { emergência da cidade de Espoo, } \\
\text { Finlândia }\end{array}$ \\
\hline 6 & $\begin{array}{l}\text { Implantação do acolhimento com } \\
\text { classificação de risco em } \\
\text { serviço hospitalar de emergência: } \\
\text { atuação do enfermeiro }\end{array}$ & $\begin{array}{l}\text { Bellucci Júnior, JA; } \\
\text { Matsuda, LM; }\end{array}$ & $\begin{array}{l}\text { Ciências, cuidado e } \\
\text { saúde }\end{array}$ & 2012 & $\begin{array}{c}\text { Relatar a atuação do enfermeiro } \\
\text { no processo de implantação do } \\
\text { acolhimento com classificação } \\
\text { de risco no serviço hospitalar } \\
\text { de emergência do Hospital } \\
\text { Universitário Regional de } \\
\text { Maringá - PR }\end{array}$ \\
\hline 7 & $\begin{array}{l}\text { Atividades do enfermeiro nos sistemas } \\
\text { de triagem/classificação } \\
\text { de risco nos serviços de urgência: } \\
\text { revisão integrativa }\end{array}$ & $\begin{array}{c}\text { Acosta, AM; } \\
\text { Duro, CLM; } \\
\text { Lima, MADDS. }\end{array}$ & $\begin{array}{l}\text { Revista Gaúcha de } \\
\text { Enfermagem }\end{array}$ & 2012 & $\begin{array}{l}\text { Identificar e avaliar as evidências } \\
\text { disponíveis na literatura sobre as } \\
\text { atividades do enfermeiro na } \\
\text { classificação de risco nos } \\
\text { serviços de urgência }\end{array}$ \\
\hline 8 & $\begin{array}{c}\text { Triage in Norwegian emergency } \\
\text { departments }\end{array}$ & $\begin{array}{l}\text { Engebretsen, S; } \\
\text { Róise, Ó; } \\
\text { Ribu, L; }\end{array}$ & $\begin{array}{l}\text { Tidsskrift for Den } \\
\text { Norske Laegeforening }\end{array}$ & 2013 & $\begin{array}{l}\text { Investigar o uso e a organização } \\
\text { da triagem, bem como o uso } \\
\text { de escalas de triagem nos } \\
\text { departamentos de emergência }\end{array}$ \\
\hline 9 & $\begin{array}{l}\text { Accuracy of triage for children with } \\
\text { chronic illness and infectious symptoms }\end{array}$ & $\begin{array}{l}\text { Seiger, N; } \\
\text { Van, VM; } \\
\text { Steyerberg, EW; } \\
\text { Lei, JVD; } \\
\text { MOLL, HA. }\end{array}$ & Pediatrics & 2013 & $\begin{array}{c}\text { Determinar a validade do } \\
\text { Sistema de Triagem de } \\
\text { Manchester em atendimento de } \\
\text { emergência para a população } \\
\text { geral }\end{array}$ \\
\hline
\end{tabular}




\begin{tabular}{|c|c|c|c|c|c|}
\hline 10 & $\begin{array}{c}\text { Análise do fluxo de atendimento de } \\
\text { serviço hospitalar de emergência: } \\
\text { estudo de caso }\end{array}$ & $\begin{array}{l}\text { Bellucci Júnior, JA; } \\
\text { Matsuda, LM; } \\
\text { Marcon, SS. }\end{array}$ & $\begin{array}{l}\text { Revista Eletrônica de } \\
\text { Enfermagem }\end{array}$ & 2015 & $\begin{array}{l}\text { Analisar o fluxo de atendimento } \\
\text { em dois Serviços } \\
\text { Hospitalares de Emergência }\end{array}$ \\
\hline 11 & $\begin{array}{c}\text { Embracement with risk classification } \\
\text { in the emergency department from } \\
\text { perspective of older adults }\end{array}$ & $\begin{array}{l}\text { Gonçalves, AVF; } \\
\text { Bierhals, CCK; } \\
\text { Paskulinc, LMG. }\end{array}$ & $\begin{array}{l}\text { Revista Gaúcha de } \\
\text { Enfermagem }\end{array}$ & 2015 & $\begin{array}{l}\text { Avaliar as práticas de } \\
\text { acolhimento na classificação de } \\
\text { risco do serviço de emergência } \\
\text { do Hospital de Clínicas de Porto } \\
\text { Alegre na perspectiva do idoso }\end{array}$ \\
\hline 12 & $\begin{array}{l}\text { Agreement between triage category and } \\
\text { patient's perception of priority in } \\
\text { emergency departments }\end{array}$ & $\begin{array}{l}\text { Toloo, GS; } \\
\text { Aitken, P; } \\
\text { Crilly, J; } \\
\text { FitzGerald, G. }\end{array}$ & $\begin{array}{l}\text { Scandinavian } \\
\text { Journal of Trauma, } \\
\text { Resuscitation and } \\
\text { Emergency Medicine }\end{array}$ & 2016 & $\begin{array}{l}\text { Compreender a extensão da } \\
\text { concordância/ discordância } \\
\text { entre os pacientes percebidos } \\
\text { prioridade e categoria de triagem } \\
\text { atual e fatores associados }\end{array}$ \\
\hline 13 & $\begin{array}{l}\text { Work process of nurses in the reception } \\
\text { with risk rating }\end{array}$ & $\begin{array}{c}\text { Rates, HF; } \\
\text { Alves, M; } \\
\text { Cavalcante, RB. }\end{array}$ & $\begin{array}{l}\text { Revista Mineira de } \\
\text { Enfermagem }\end{array}$ & 2016 & $\begin{array}{c}\text { Descrever o processo de } \\
\text { trabalho do enfermeiro no } \\
\text { Acolhimento com Classificação } \\
\text { de Risco na Unidade de Pronto } \\
\text { Atendimento } \\
\text { de um município de Minas } \\
\text { Gerais }\end{array}$ \\
\hline 14 & $\begin{array}{l}\text { Percepção dos usuários sobre a triagem } \\
\text { com classificação de } \\
\text { risco em um serviço de urgência de } \\
\text { Cabo Verde }\end{array}$ & $\begin{array}{l}\text { Spagnuolo, RS; } \\
\text { Silva, MNL; } \\
\text { Meneguin, S; } \\
\text { Bassetto, JGB; } \\
\text { Fernandes, VC. }\end{array}$ & $\begin{array}{l}\text { Revista Brasileira em } \\
\text { Promoção da Saúde }\end{array}$ & 2017 & $\begin{array}{l}\text { Desvelar as concepções dos } \\
\text { usuários acerca da triagem com } \\
\text { classificação de risco em um } \\
\text { serviço de urgência }\end{array}$ \\
\hline 15 & $\begin{array}{c}\text { Manchester protocol and user } \\
\text { population in the risk assessment: the } \\
\text { nurse's view }\end{array}$ & $\begin{array}{l}\text { Roncalli, AA; } \\
\text { Oliveira, DND; } \\
\text { Silva, ICM; } \\
\text { Brito, RF; } \\
\text { Viegas, SMDF. }\end{array}$ & $\begin{array}{l}\text { Revista Baiana de } \\
\text { Enfermagem }\end{array}$ & 2017 & $\begin{array}{l}\text { Compreender a visão do } \\
\text { enfermeiro sobre a utilização } \\
\text { do Protocolo de Manchester } \\
\text { e a população usuária na } \\
\text { classificação de risco de uma } \\
\text { Unidade de Pronto Atendimento }\end{array}$ \\
\hline 16 & $\begin{array}{l}\text { Everyday experiences of the nurse in } \\
\text { risk classification in an emergency unit }\end{array}$ & $\begin{array}{l}\text { Roncalli, AA; } \\
\text { Oliveira, DND; } \\
\text { Melo, IC; } \\
\text { Viega, SMDF; } \\
\text { Brito, RF. }\end{array}$ & $\begin{array}{l}\text { Revista de } \\
\text { Enfermagem UFPE } \\
\text { Online }\end{array}$ & 2017 & $\begin{array}{l}\text { Compreender a vivência } \\
\text { do enfermeiro que atua na } \\
\text { classificação de risco de uma } \\
\text { Unidade de Pronto } \\
\text { Atendimento }\end{array}$ \\
\hline 17 & $\begin{array}{l}\text { Accuracy of emergency department } \\
\text { triage using the Emergency Severity } \\
\text { Index and independent predictors } \\
\text { of under-triage and over-triage: a } \\
\text { retrospective cohort analysis. }\end{array}$ & $\begin{array}{c}\text { Hinson, JS; } \\
\text { Martinez, DA; } \\
\text { Schmitz, PSK; } \\
\text { Toerper, M; } \\
\text { Radu, D; } \\
\text { Scheulen, J; } \\
\text { Ramirez, SAS; } \\
\text { Levin, S. }\end{array}$ & $\begin{array}{l}\text { International Journal of } \\
\text { Emergency Medicine }\end{array}$ & 2018 & $\begin{array}{l}\text { Mensurar a frequência de sub ou } \\
\text { super classificação de pacientes } \\
\text { triados por enfermeiros } \\
\text { usando o índice de gravidade } \\
\text { de emergência no Brasil e } \\
\text { identificar os fatores associados }\end{array}$ \\
\hline 18 & $\begin{array}{l}\text { Evaluation of the triage performed by } \\
\text { registered nurses in the Hospital Clínico } \\
\text { Universitario Lozano Blesa Emergency } \\
\text { Service }\end{array}$ & $\begin{array}{l}\text { Viñuales, I; } \\
\text { Monzón-Fernandez, A; } \\
\text { Viñuales, M; } \\
\text { Sanclemente, T. }\end{array}$ & Enfermería Clínica & 2018 & $\begin{array}{l}\text { Avaliar a qualidade e adequação } \\
\text { da triagem realizada por } \\
\text { enfermeiras no Hospital Clínico } \\
\text { de Saragossa e comparar } \\
\text { as características das visitas } \\
\text { urgentes e não urgentes }\end{array}$ \\
\hline
\end{tabular}

Fonte: dados da pesquisa, 2018.

Quanto ao idioma das publicações, verificaram-se cinco $(27,8 \%)$ artigos redigidos exclusivamente em língua portuguesa, quatro $(22,2 \%)$ com versões disponíveis em língua portuguesa e inglesa, oito $(44,5 \%)$ exclusivamente em língua inglesa e um (5,5\%) exclusivamente em língua espanhola. Dos 18 artigos, nove (50\%) foram indexados na base de dados MEDLINE, seis $(33,3 \%)$ na LILACS e três (I6,7\%) na BDENF, conforme tabela I.
Com relação aos periódicos, não foi evidenciada predominância de publicações em um periódico específico, ou seja, os artigos foram publicados em períodos variados. Com relação ao ano das publicações, houve variação de um a três artigos por ano, com exceção dos anos 2009 e 2014 , nos quais não foram identificados artigos que respondessem à questão norteadora deste estudo. No que se refere à classificação qualis, nove $(60 \%)$ 
TABELA 1 - Distribuição dos artigos de acordo com as bases de dados. Rio Grande (RS), Brasil, 2018.

\begin{tabular}{c|c|c}
\hline Bases de Dados & Frequência & \% \\
\hline MEDLINE & 09 & $50 \%$ \\
\hline LILACS & 06 & $33,3 \%$ \\
\hline BDENF & 03 & $16,7 \%$ \\
\hline Total & $\mathbf{1 6}$ & $\mathbf{1 0 0 \%}$ \\
\hline
\end{tabular}

Fonte: dados da pesquisa, 2018.

dos periódicos possuíam classificação e seis (40\%) não possuíam. Daqueles que a tinham, quatro $(44,5 \%)$ eram classificados como qualis $\mathrm{BI}$, três $(33,3 \%)$ como B2, um (II, I\%) como B3 e um como A2 (II,I\%).

No que diz respeito ao nível de evidência dos artigos, 16 (88,9\%) foram classificados no nível Vl, indicando evidências provenientes de estudos qualitativos e/ou descritivos. Os dois (I I, I\%) restantes classificaram-se no nível V, revelando evidências provenientes de revisões sistemáticas ${ }^{16}$.

A partir da Análise de Conteúdo foram identificadas três categorias: o enfermeiro no contexto da classificação de risco, capacitação do enfermeiro atuante na classificação de risco e atuação do enfermeiro no contexto da classificação de risco. Por meio desta categorização, foi possível entender como se dá a atuação do enfermeiro na $C R$ de SUE, bem como identificar os fatores intervenientes.

\section{DISCUSSÃO}

Percebeu-se uma heterogeneidade de estudos a respeito da atuação do enfermeiro na CR dos SUE, bem como uma leve tendência de crescimento no que se refere à produção nacional acerca desta temática. Identificou-se como limitação do estudo a grande variedade de descritores e palavras-chave que são utilizadas como sinônimas do termo "classificação de risco". Em decorrência disso, pode haver interferência na identificação do número real de publicações disponíveis acerca da temática.

Alguns dos resultados encontrados foram de encontro aos achados de pesquisa nacional que objetivou identificar e avaliar as evidências disponíveis na literatura a respeito das atividades do enfermeiro na $C R$ dos serviços de urgência, no recorte temporal de 2000 a $2010^{17}$. Contudo, em comparação com a pesquisa supracitada, o presente estudo verificou que, embora não tenha havido um crescimento no número total de produções acerca dessa temática, houve um aumento no número de estudos nacionais. Enquanto que no recorte temporal de 2000 a 2010 identificaram-se dois estudos nacionais sobre o tema ${ }^{17}$, no período que compreende 2008 a 2018 encontraram-se seis artigos nacionais, conforme apresentado nos resultados deste estudo.Além disso, depararam-se novos achados, os quais serão discutidos na sequência.

\section{O enfermeiro no contexto da classificação de risco}

O processo de trabalho na CR dos SUE envolve atuação multiprofissional e multidisciplinar, incluindo recep- cionistas, equipe de enfermagem, equipe médica, equipe laboratorial, equipe de radiologia e outras ${ }^{18}$. No entanto, o protagonista da CR é o enfermeiro ${ }^{11,19,20}$.

Em países como Finlândia, França, Holanda, Noruega, Austrália e Brasil, o enfermeiro é o responsável pela realização da $C R^{19,21-26}$. Sua linguagem clínica orientada para queixas, sinais e sintomas, favorece a identificação do estado geral do paciente e da gravidade ou não de seu quadro clínico. Além disso, outras características do enfermeiro contribuem para sua atuação no contexto da CR. São elas: empatia e visão global do serviço e dos recursos nele existentes ${ }^{27}$.

A empatia é uma característica fundamental na minimização de sentimentos como ansiedade, agressividade ou impaciência, frequentemente encontrados em pacientes atendidos na CR dos SUE.A minimização desses sentimentos evita atrasos no fluxo do serviço, conflitos entre os membros da equipe de saúde e os pacientes e, até mesmo, agravamento do quadro clínico dos pacientes ${ }^{27,18}$.

Já a característica da visão global do serviço e dos recursos nele existentes permite a identificação do suporte e dos cuidados que podem ser ofertados aos pacientes, de forma a agilizar o atendimento ${ }^{27}$. Estudos nacionais apontaram que o desconhecimento acerca da rotina dos setores de exames laboratoriais e de imagem, associado à falta de comunicação entre as equipes médica e de enfermagem resultou em aumento no fluxo de pacientes nos SUE e em atrasos no diagnóstico e tratamento daqueles que passaram pela $C R^{27,28}$.

Ademais, para que consiga avaliar o estado geral do paciente, identificar os fatores associados ao seu quadro clínico e classificar sua prioridade de atendimento, o enfermeiro necessita das algumas habilidades. São elas: escuta qualificada, raciocínio clínico e agilidade para tomada de decisões, avaliação e detalhamento das queixas e conhecimento da rede assistencial para os encaminhamentos necessários ${ }^{18}$.

A escuta qualificada propicia a construção do vínculo terapêutico e, consequentemente, garante $o$ acesso a dados subjetivos e objetivos do paciente. Quanto mais detaIhada a investigação dos dados, mais precisa é a classificação. Em posse desses dados, o enfermeiro utiliza-se de seu raciocínio clínico para determinar a categoria de urgência do paciente e se há necessidade de encaminhamentos ${ }^{29,9}$.

Por fim, é importante que o enfermeiro atuante na CR tenha conhecimentos acerca das Redes de Atenção à Saúde. Esse conhecimento facilita o encaminhamen- 
to/contrarreferenciamento dos pacientes com quadros clínicos de baixa complexidade aos serviços adequados para a solução de suas necessidades de saúde ${ }^{30,28}$.

\section{Capacitação do enfermeiro atuante na classificação de risco}

Verificou-se que, para atuar na CR, o enfermeiro necessita ser capacitado. Geralmente, as instituições fornecem capacitações aos profissionais contratados para atuar no setor.Além disso, as especializações/qualificações e o tempo de experiência na área são fatores que contribuem para o desenvolvimento profissional do enfermeiro na CR.

Estudos europeus constataram que, ao serem contratados para atuar na CR dos SUE, os profissionais foram capacitados, a fim de evitar erros de classificação ${ }^{24,19}$. Em consonância, estudo brasileiro revelou que, previamente à implantação da CR nos SUE de Maringá - Paraná, os enfermeiros foram capacitados para atuar no setor por meio de oficinas e de visitas técnicas a instituições locais que já haviam adotado protocolos de $C R^{31}$.

No entanto, as capacitações oferecidas pelos contratantes podem não ser suficientes para subsidiar o trabaIho do enfermeiro na CR. Nesse sentido, pesquisa efetuada em Belo Horizonte - Minas Gerais apontou que, embora tenham sido capacitados acerca da CR pela instituição em que trabalham, os enfermeiros de um Serviço de Pronto Atendimento consideraram a capacitação recebida insuficiente para subsidiar a prática de classificação dos riscos clínicos. Eles atribuíram esse posicionamento a curta duração (um dia) da capacitação fornecida e ao fato de a $C R$ não ser um conteúdo abordado durante a Graduação em Enfermagem. Afora isso, o estudo desvelou que a atualização dos saberes técnico-científicos e as experiências vivenciadas no cotidiano de trabalho são imprescindíveis para a construção do conhecimento necessário para subsidiar as ações na $\mathrm{CR}^{26}$.

Quando realizada por profissionais não capacitados, a $C R$ pode ser equivocada. $O$ resultado dessa ação inadequada são os erros de classificação e, consequentemente, a ocorrência de eventos adversos para os pacientes e para os SUE.

Estudos internacionais e nacionais ratificaram esse achado e desvelaram que os principais erros na $C R$ dos SUE são a subtriagem ou classificação subestimada e a hipertriagem ou classificação hiperestimada ${ }^{23,7}$. A classificação subestimada acontece quando a queixa do paciente é desvalorizada pelo classificador, de maneira que o mesmo é classificado em níveis de gravidade abaixo do indicado para seu quadro clínico. Já a classificação superestimada incide quando a queixa do paciente é supervalorizada pelo classificador, de modo que o mesmo é classificado em níveis de gravidade acima do adequado para seu quadro clínico ${ }^{7}$.
Esses erros de classificação podem implicar em consequências sérias para os pacientes e os SUE.A classificação subestimada está associada à elevação nos índices de eventos adversos ao paciente e ao aumento no número de internações de pacientes em estado crítico. Já a classificação superestimada, embora seja um erro associado à maior segurança para o paciente, correlaciona-se ao aumento na demanda de pacientes não urgentes nos SUE, consumindo recursos de saúde necessários ao atendimento daqueles que se encontram em quadros clínicos graves. As consequências desses erros tornam-se ainda mais graves na classificação de pacientes pediátricos ${ }^{7,32,23}$.

Os erros de classificação ocorrem, principalmente, quando o enfermeiro classificador não é capacitado para desempenhar esse papel. Somadas à falta de capacitação, a presença de queixas e sintomas tidos como "fatores confundidores" e de doenças crônicas podem contribuir para a ocorrência desses erros.

Estudo que objetivou identificar e mensurar os erros de classificação verificou que algumas queixas e sintomas apresentados pelos pacientes favoreceram a classificação subestimada ou superestimada. Foram queixas preditivas de classificação subestimada: idade avançada, bradicardia, taquicardia, hipóxia, hipertermia e sintomas como dor torácica e dispnéia. Quanto às queixas preditivas da classificação superestimada, foram nomeados os quadros hipertensivos e alérgicos ${ }^{7}$. Paralelamente, estudo holandês que investigou a acurácia da CR de crianças com doenças crônicas e sintomas infecciosos, desvelou que a precisão na classificação foi maior nas crianças com sintomas infecciosos que não possuíam doenças crônicas, do que nas crianças com quadro clínico semelhante, porém com doenças crônicas ${ }^{23}$.

Frente à existência de todos estes fatores intervenientes na $\mathrm{CR}$, torna-se essencial ao enfermeiro um amplo conhecimento técnico-científico e o desenvolvimento de pensamento crítico e raciocínio clínico, além de capacitações e atualizações a respeito das técnicas e protocolos utilizados para a CR.Ainda, faz-se importante a visão holística do paciente, de modo que seja considerada sua diversidade, individualidade e singularidade. Isso permite evitar os erros de classificação e, consequentemente, a ocorrência de eventos adversos aos pacientes, sejam eles adultos ou pediátricos ${ }^{33}$.

\section{Atuação do enfermeiro na classificação de risco}

Identificou-se que a atuação do enfermeiro na CR perpassa as esferas da avaliação clínica, da tomada de decisão, do monitoramento, do registro, do gerenciamento, da educação e da resolução e/ou encaminhamento dos pacientes.

No que se refere à avaliação clínica, por meio da consulta de enfermagem, o enfermeiro realiza uma breve avaliação dos pacientes, composta por anamnese e exa- 
me físico, voltados a sua queixa principal. Com base nos dados subjetivos e objetivos colhidos durante a avaliação, e em seu conhecimento clínico, o enfermeiro efetua a tomada de decisão acerca da priorização do atendimento. Isto é, o enfermeiro articula seus instrumentos e saberes científicos às queixas, sinais e sintomas manifestados pelos pacientes e, através de seu raciocínio clínico, atribui uma categoria de risco ao paciente $e^{25,26,20}$.

A tomada de decisão na CR é amparada por meio de protocolos, visto que a classificação subjetiva é inespecífica e mais sujeita a erros ${ }^{34}$. No entanto, o enfermeiro não deve uniformizar totalmente $\mathrm{o}$ atendimento, e sim atentar para as particularidades e aspectos individuais e singulares de cada paciente atendido. Caso contrário, pode-se ter uma CR subestimada ${ }^{23}$. Estudos apontaram que as decisões tomadas pelos enfermeiros na $C R$ devem ser precisas, visto que envolvem a diferenciação entre pacientes que podem ou não esperar pelo atendimento médico e a determinação da dinâmica de trabalho nos SUE ${ }^{17,22,25}$.

Após avaliar e classificar os pacientes, é importante que o enfermeiro monitore-os enquanto aguardam pela consulta médica. Esse monitoramento visa a identificação de situações, como dor ou fome, que podem ser manejadas pelo enfermeiro, de acordo com protocolos institucionais. Nesse sentido, estudo realizado em um SUE localizado no sul do Brasil evidenciou que a instituição possuía um protocolo, no qual estava prevista a analgesia de pacientes já classificados, que aguardavam a consulta médica. Isso é importante, principalmente, quando se trata de pacientes idosos, nos quais as queixas álgicas aumentam e se intensificam progressivamente, devido às alterações fisiopatológicas típicas desses indivíduos ${ }^{33}$. A identificação e o manejo dessas situações representam ações de humanização do cuidado na CR dos SUE.

Toda a assistência prestada ao paciente na CR, desde sua recepção até a alta, deve ser devidamente registrada em formulários ou sistemas eletrônicos, de acordo com a rotina da instituição. $O$ enfermeiro é o responsável pelo registro de dados referentes aos sinais vitais, ao histórico de saúde, às intervenções diagnósticas e terapêuticas, à admissão hospitalar e à evolução do quadro clínico dos pacientes. Esses registros são essenciais tanto para garantir a segurança dos pacientes, quanto assegurar o respaldo legal dos profissionais e dos SUE ${ }^{34}$.

O gerenciamento é um dos subprocessos que compõe o trabalho do enfermeiro na CR. É esse profissional quem gerencia a demanda/fluxo de atendimentos, a classificação e o cuidado aos pacientes, os recursos financeiros destinados ao setor, os recursos humanos, a educação permanente da equipe de enfermagem e os conflitos interpessoais entre a equipe de saúde ${ }^{31,26}$. Nesse contexto, a liderança é uma característica importante para que o enfermeiro desenvolva suas atividades gerenciais, de modo a alcançar qualidade no atendimento, bem-estar dos pacientes e o desenvolvimento profissional da equipe multidisciplinar que atua na $\mathrm{CR}^{31,35-37}$.

Para que as atividades realizadas pelo enfermeiro na CR sejam seguras e de qualidade, é necessária a educação permanente. $O$ saber funciona como uma extensão do profissional, permitindo a condução do cuidado ${ }^{20,18}$. Estudo mineiro revelou que o conhecimento científico é um elemento indispensável para que a prática do enfermeiro na $C R$ não seja mecanizada. $O$ enfermeiro que domina os conhecimentos científicos não apenas classifica os riscos clínicos dos pacientes, mas, também, assiste-os integralmente, em sua singularidade ${ }^{26}$.

O conhecimento acerca das rotinas do SUE também é imprescindível para a atuação do enfermeiro na CR. Estudo executado em Porto Alegre - Rio Grande do Sul verificou que o desconhecimento sobre o funcionamento da CR e o sentimento de "estar perdido" são frequentes entre os pacientes que recorrem aos SUE. Nesse sentido, o enfermeiro deve orientá-los a respeito da finalidade, do funcionamento, do fluxo e do tempo de atendimento no serviço, evitando, assim, ansiedade e piora do quadro clínico dos pacientes ${ }^{33}$.

Ao final do atendimento na $C R$, o enfermeiro tem duas opções: dar resolubilidade às necessidades apresentadas pelo paciente no atendimento inicial ou o encaminhá-lo para o atendimento/serviço mais adequado. Portanto, ao classificar os pacientes, o enfermeiro deve garantir que aqueles classificados como urgentes passem pela consulta médica para diagnóstico e prescrição da terapêutica adequada, e os que foram classificados como não urgentes sejam encaminhados à Atenção Básica para que suas necessidades sejam sanadas ${ }^{21}$.

Estudos nacionais realizados em Belo Horizonte, Florianópolis e Londrina evidenciaram que muitos pacientes atendidos na CR dos SUE foram encaminhados à Atenção Básica, devido a não urgência de seus quadros clínicos ou para continuidade da assistência ${ }^{21,26,37}$. No entanto, alguns estudos apontaram que não existe um contrarreferenciamento eficiente entre os SUE e a Atenção Básica. Essa falta de comunicação entre os serviços da rede de atenção à saúde representa um risco de comprometimento à segurança do paciente. Além disso, ocasiona superlotação e interferências na assistência integral aos pacientes nos SUE ${ }^{30,28}$.

\section{CONCLUSÃO}

No que se refere à atuação do enfermeiro na CR dos SUE, identificou-se sua participação ativa na assistência ao paciente, na tomada de decisões, no monitoramento dos pacientes atendidos, no registro das informações inerentes ao atendimento, no gerenciamento, na educação e na resolubilidade dos problemas dos pacientes e/ou 
encaminhamento dos mesmos aos serviços adequados. Ademais, constatou-se que, para o desenvolvimento de tais atividades, o enfermeiro necessita de conhecimento técnico-científico, escuta qualificada, raciocínio clínico, agilidade para tomada de decisões e conhecimento da rede assistencial, a fim de prestar um atendimento seguro e de qualidade.
Espera-se contribuir para compreensão da atuação do enfermeiro na CR dos SUE. Sugerem-se novas pesquisas a respeito da capacitação dos enfermeiros para o desenvolvimento da CR, da implementação de atividades de educação permanente relacionadas a essa temática e da abordagem da CR na grade curricular dos Cursos de Graduação em Enfermagem. 


\section{REFERÊNCIAS}

I.Gloss K. End of life care in emergency departments: a review of literature. Emerg Nurse [Internet]. 2017 [acesso em 20 fev 20l8]; 25(2):29-36. Disponível em: https://journals.rcni. com/emergency-nurse/end-of-life-care-in-emergency-departments-a-review-of-the-literature-aop-en.2017.el707

2. Viñuales I, Monzón-Fernández A, Viñuales M, Sanclemente $\mathrm{T}$. Evaluation of the triage performed by registered nurses in the hospital clinic universitario Lazano Blessa Emergency Service (Saragossa, Spain). Enferm Clin [Internet]. 2018 [acesso em 20 fev 20I8]; 28(2):I-5. Disponível em: http:// www.elsevier.es/es-revista-enfermeria-clinica-35-linkresolver-evaluacion-del-triaje-realizado-por-S I I 30862 I I 730 I 936

3. Yarmohammadian MH, Rezaei F, Haghshenas A, Tavakoli N. Overcrowding in emergency departments: a review of strategies to decrease future challenges.J Res Med Sci [Internet]. 2017 [acesso em 20 fev 20I8]; 22(23): I-2I. Disponível em: https://www.ncbi.nlm.nih.gov/pmc/articles/PMC5377968/

4. Jobé J, Donneau AF, Scholtes B, Ghuysen A. Quantifying emergency department crowding: comparison between two scores. Acta Clin Belg [Internet]. 2017 [acesso em $20 \mathrm{fev}$ 2018]; 33(22):I-6. Disponível em: https://www.ncbi.nlm.nih. gov/pubmed/29207925

5. Chiu IM, Lyn YR, Syue YJ, Kung CT, Wu KH, Li CJ. The influence of crowding on clinical practice in the emergency department. Am J Med [Internet]. 2018 [acesso em $20 \mathrm{fev}$ 2018]; 36(I):56-60. Disponível em: http://www.ajemjournal. com/article/S0735-6757(17)30539-9/fulltext

6. Brasil. Ministério da Saúde. Política Nacional de Humanização: a humanização como eixo norteador das práticas de atenção e gestão em todas as instâncias do SUS. Brasília: Ministério da Saúde [Internet]; 2004 [acesso em 20 fev 2018]. Disponível em: http://bvsms.saude.gov.br/bvs/publicacoes/ humanizasus_2004.pdf

7. Hinson JS, Martinez DA, Scmitz PSK, Toerper M, Radu D, Scheulen J, et al. Accuracy of emergency department triage using the Emergency Severity Index and independent predictors of under-triage and over-triage in Brazil: a retrospective cohort analysis. Int J Emerg Med [Internet]. 2018 [acesso em 20 fev 20I8]; II(3):I-I0. Disponível em: https://intjem. springeropen.com/articles/ I0.I I86/s I 2245-0 I7-0 I6I-8

8. Soler W, Gómez MM, Braqulat E, Alvares A. El triaje: herramienta fundamental en urgencias y emergencias. An Sis Sanit Navar [Internet]. 2010 [acesso em 20 fev 20l8]; 33(Suppl I):55-68. Disponível em: http://scielo.isciii.es/pdf/asisna/ v33s I/original8.pdf

9. Souza CCD. Nurse's decision-making process on risk classification: what is the theoretical basis for this practice in the health care scenario?. Rev Min Enferm [Internet]. 2015 [acesso em 20 fev 2018]; 19(2): 12-3. Disponível em: http:// www.reme.org.br/artigo/detalhes/l00 I

10. Mirhaghi A, Ebrahimi M. The Australasian Triage Scale Level 5 Criteria May Need to be revised: a commentary.
Emerg [Internet]. 2017 [acesso em 20 fev 20।8]; 5(I):I-2. Disponível em: https://www.ncbi.nlm.nih.gov/pmc/articles/ PMC5325922/pdf/emerg-5-e50.pdf

I I. Leite L, Baptista R, Leitão J, Cochicho J, Breda F, Elvas L, et al. Chest pain in the emergency department: risk stratification with Manchester triage system and HEART score. BMC Cardiovasc Disord [Internet]. 2015 [acesso em 20 fev 20।8]; I5(48): I-7. Disponível em: https://www.ncbi.nlm. nih.gov/pmc/articles/PMC4462 I |4/pdf/ 12872_20I5_Article_49.pdf

12. Conselho Federal De Enfermagem. Resolução COFEN $n^{\circ} 423$, de 9 de abril de 20I2. Normatiza, no âmbito do Sistema COFEN/Conselhos Regionais de Enfermagem, a participação do enfermeiro na classificação de risco. Brasília: COFEN [Internet]; 2012 [acesso em 20 fev 20l8]. Disponível em: http://www.cofen.gov.br/resoluo-cofen -n-42320I2_8956.html

13. Conselho Federal De Enfermagem. Resolução $n^{\circ} 358$, de 15 de outubro de 2009. Dispõe sobre a sistematização da assistência de enfermagem e a implementação do processo de enfermagem em ambientes, públicos ou privados, em que ocorre o cuidado de enfermagem e dá outras providências. Brasília: COFEN [Internet]; 2009 [acesso em 20 fev 20 I8]. Disponível em: http://www.cofen.gov.br/resoluocofen-3582009_4384.html

14. Whittemore R, Knafl K. The integrative review: updated methodology. J Adv Nurs [Internet]. 2005 [acesso em 20 fev 20 18];52(5):546-53. Disponível em: https://onlinelibrary. wiley.com/doi/abs/ I 0. I I I I/j. I 365-2648.2005.0362 I.x

I5. Bardin L.Análise de Conteúdo. São Paulo: Edições 70; 201 I. 16. Melnyk BM, Fineout-Overholt E. Evidence based practice in nurse and healthcare: a guide to best practice. 2th ed. Philadelphia:Wolters Kluwer; $201 \mathrm{I}$.

17. Acosta AM, Duro CLM, Lima MADDS.Atividades do enfermeiro nos sistemas de triagem/classificação de risco nos serviços de urgência: revisão integrativa. Rev Gaúcha Enferm [Internet]. 2012 [acesso em 29 mar 20l8]; 33(4): I8I90. Disponível em: http://www.scielo.br/pdf/rgenf/v33n4/ en_23.pdf

18. Roncalli AA, Oliveira DND, Melo IC, Viegas SMDF, Brito RF. Everyday experiences of the nurse in risk classification in an emergency unit. Rev Enferm UFPE Online [Internet]. 2017 [acesso em 20 fev 20I8]; I (4): I743-5I. Disponível em: https://periodicos.ufpe.br/revistas/revistaenfermagem/ article/view/I5246/18029

19. Kantonen J, Menezes R, Heinanen T, Mattila J, Matilla KJ, Kaupilla T. Impact of the ABCDE triage in primary care emergency department on the number of patient visits to different parts of the health care system in Espoo City. BMC Emerg Med [Internet]. 2012 [acesso em 20 fev 20 I8]; I2(2):I-12. Disponível em: https://www.ncbi.nlm.nih.gov/ pmc/articles/PMC3267646/

20. Rates HF, Alves M, Cavalcante RB. Work process of nurses 
in the reception with risk rating. Rev Min Enferm [Internet]. 2016 [acesso em 20 fev 20l8]; 20(I): I-6. Disponível em: http://reme.org.br/artigo/detalhes/I 105

2I. Rossaneis MA, Haddad MDCL, Borsato FG, Vannuchi MO, Sentone ADD. Caracterização do atendimento após implantação do acolhimento, avaliação e classificação de risco em hospital público. Rev Eletr Enferm [Internet]. 20I I [acesso em 20 fev 2018]; I3(4):648-56. Disponível em: https://www.fen.ufg.br/revista/v13/n4/pdf/v13n4a08.pdf

22. Durand AC, Gentile S, Gerbeaux P,Alazia M, Kiegel P, Luigi $S$, et al. Be careful with triage in emergency departments: interobserver agreement on I,578 patients in France. BMC Emerg Med [Internet]. 20II [acesso em 20 fev 20l8]; II(I9):I-I0. Disponível em: https://www.ncbi.nlm.nih.gov/ pmc/articles/PMC3215166/

23. Seiger N, Veen MV, Steyerberg EW, Lei JVD, Moll HA. Accuracy of triage for children with chronic illness and infectious symptoms. Pediatrics [Internet]. 2013 [acesso em 20 fev 2018]; 132(6):I-9. Disponível em: http://pediatrics. aappublications.org/content/ /32/6/e 1602

24. Engebretsen S, Roise O, Ribu L. Triage in Norwegian emergency departments. Tidsskr Nor Laegeforen [Internet]. 2013 [acesso em $20 \mathrm{fev} 2018$ ]; 133(3):285-9. Disponível em: https://tidsskriftet.no/en/2013/02/triage-norwegian-emergency-departments

25. Toloo GS, Aitken P, Crilly J, Fitzgerald G. Agreement between triage category and patient's perception of priority in emergency departments. Scand J Trauma Resusc Emerg Med [Internet]. 2016 [acesso em 20 fev 20I8]; 24(I):I-8. Disponível em: https:/www.ncbi.nlm.nih.gov/pmc/articles/ PMC5070359/pdf/I3049_2016_Article_316.pdf

26. Roncalli AA, Oliveira DND, Silva ICM, Brito RF, Viegas SMDF. Manchester protocol and user population in the risk assessment: the nurse's view. Rev Baiana Enferm [Internet]. 2017 [acesso em 20 fev 20 I8]; 3 I (2): I-I0. Disponível em: https://portalseer.ufba.br/index.php/enfermagem/article/ view/I6949//45/2

27. Spagnuolo RS, Silva MNL, Meneguin S, Bassetto JGB, Fernandes VC. Users perception of the risk assessment screening in an emergency care service in Cape Verde. Rev Bras Promoç Saúde [Internet]. 2017 [acesso em 20 fev 20l8]; 30(2):249-54. Disponível em: http://periodicos.unifor.br/ RBPS/article/view/5845/pdf_I

28. Bellucci Júnior JA, Matsuda LM, Marcon SS.Análise do fluxo de atendimento de serviço hospitalar em emergência: estudo de caso. Rev Eletr Enferm [Internet]. 2015 [acesso em 29 mar 2018]; I7(I):I08-16. Disponível em: https://www. fen.ufg.br/revista/vI7/nI/pdf/vI7nla I3.pdf

29. Souza MMT, Passos JP. O trabalho do enfermeiro no con- texto hospitalar sob a perspectiva da clínica da atividade: nota prévia. Enferm Atual [Internet]. 2018 [acesso em 29 mar 20।8]; I8(84):195-6. Disponível em: http://inderme. com.br/revista/22/revista.pdf

30. Costa MAR,Versa GLGS, Bellucci Júnior JA, Inoue KC, Sales CA, Matsuda LM.Admittance of risk-classified cases: assessment oh hospital emergency services. Esc Anna Nery [Internet]. 2015 [acesso em 29 mar 20I8]; 19(3):49I-7. Disponível em: http://www.scielo.br/pdf/ean/v19n3/en_|4|48|45-ean-19-03-049I.pdf

31. Bellucci Júnior JA, Matsuda LM. Implantação do acolhimento com classificação de risco em serviço hospitalar de emergência: atuação do enfermeiro. Ciênc Cuid Saúde [Internet]. 2012 [acesso em 29 mar 20I8]; I I(2):396-40I. Disponível em: http://ojs.uem.br/ojs/index.php/CiencCuidSaude/article/viewFile/ I 4922/pdf

32. Coutinho AAP, Cecílio LCDO, Mota JAC. Classificação de risco em serviços de emergência: uma discussão da literatura sobre o sistema de triagem de Manchester. Rev Med Minas Gerais [Internet]. 2012 [acesso em 29 mar 20।8]; 22(2): I8898. Disponível em: http://www.rmmg.org/artigo/detalhes/I0 I

33. Gonçalves AVF, Bierhals CCK, Paskulin LMG. Embracement with risk classification in the emergency department from perspective of olders adults. Rev Gaúcha Enferm [Internet]. 2015 [acesso em 29 mar 2018]; 36(3):14-20. Disponível em: http://seer.ufrgs.br/index.php/RevistaGauchadeEnfermagem/article/view/52422/35042

34. Veen MV, Steyerberg EW, Ruige M, Meurs AHJV, Roukema J, Lei JVD, et al. Manchester triage system in pediatric emergency care: prospective observational study. BM] [Internet]. 2008 [acesso em 29 mar 2018]; 12(7): I-7. Disponível em: https://www.ncbi.nlm.nih.gov/pmc/articles/PMC2548283/

35. Mckibben L. Conflict management: importance and implications. Br J Nurs [Internet]. 2017 [acesso em 29 mar 2018]; 26(2): I00-4. Disponível em: https://www.magonlinelibrary. com/doi/abs/I 0.12968/bjon.20 17.26.2. I00?url_ver=Z39. 88-2003\&rfr_id=ori\%3Arid\%3Acrossref.org\&rfr_dat $=c r$ pub\%3Dpubmed

36. Pinhatti EDG, Vannuchi MTO, Sardinha DDSS, Haddad MDCL. Job rotation of nursing professionals among the sectors of a hospital: a management tool in conflict resolution. Texto Contexto Enferm [Internet]. 2017 [acesso em 29 mar 2018]; 26(2): I-9. Disponível em: http://www.scielo. br/pdf/tce/v26n2/0104-0707-tce-26-02-el I800I5.pdf

37. Cassettari SDSR, Mello ALSFD. Demand and type of care provided in emergency services in the city of Florianópolis, Brazil. Texto Contexto Enferm [Internet]. 2017 [acesso em 29 mar 2018]; 26(I): I-9. Disponível em: http://www.scielo. br/pdf/tce/v26n I/I980-265X-tce-26-0 I-e34000 I5.pdf 